\title{
Towards Improving Information Systems Interoperability in Disaster Management
}

\author{
Ovidiu Noran \\ Griffith University Australia, School of ICT, O.Noran@griffith.edu.au
}

\begin{abstract}
Climate change-triggered catastrophic events appear to be steadily increasing in intensity and frequency. Proper preparation, response and recovery are essential in order to survive and recover from disasters. However, for historical, geographical and cultural reasons, the organizations responsible for delivering emergency response services often under-perform, with the lack of proper interoperation and collaboration of their information systems being a main culprit. This paper analyses interoperability issues specific to disaster management from an information systems perspective and proposes improvements based on advances in information systems and interoperability research, using an enterprise architecture perspective in order to provide a sustainable holistic and life cycle-based solution.
\end{abstract}

\section{Introduction}

History has shown that business survival and success requires a multi-pronged approach towards sustainability, reflecting Elkington's [1] Triple Bottom Line: one must achieve not only economic bottom-line performance but also environmental and social accomplishment. Blackburn [2] compares economic sustainability to air and environmental and social sustainability to food: the first is more urgent but not more important than the second. - hence, a successful enterprise must take a whole-system approach to sustainable development [3, 4].

Although emphasis on environmental sustainability is increasing worldwide, the results will take significant time to impact on and hopefully revert the current bleak environmental trends. Climate change is nowadays seen as real and the main cause of an increasing number of disasters of growing intensity. In this context, it is essential to be able to promptly and effectively prevent, prepare for, respond to and recover from catastrophic events. Governments typically respond by creating policies and agencies / organisations to tackle these aspects. The 'disaster management organisations' (DMOs) thus created operate in a complex environment (history / traditions, geographic location and culture, level of governance etc), that often promotes heterogeneity and independent evolution. Organisational diversity, while potentially beneficial, requires additional effort to achieve proper and effective collaboration [5]. In addition, coping with large scale catastrophic events usu- 


\section{Ovidiu Noran}

ally requires resources and capabilities beyond those of individual organisations, making effective cooperation and interoperation of DMOs essential. Failure to meet these requirements at all necessary levels and addressing all relevant aspects has dire consequences that typically include loss of property and even human life.

As an essential and ubiquitous component of every organisation [6], the information system (IS) plays an essential role in achieving cooperation and interoperability and thus organisational sustainability of the disaster management effort. This paper aims to focus on the IS aspect of disaster management interoperability and to propose ways to address them using knowledge accumulated in the IS and interoperability research areas. The analysis is performed from an enterprise architecture (EA) stance in an attempt to promote a whole-system and life cycle-based approach covering all aspects deemed as relevant for the disaster management universe of discourse.

\section{Current Issues in Disaster Management}

The operation of emergency services is typically legislated at state, national and international levels (see [7-10]). However, simply instructing organisations to cooperate using high-level generic directives does not bring about true collaboration and/or interoperability. The consequences are increased response times, confusion about the situation on the ground, dispute as to who, where and when is in charge. It is also difficult to coordinate with other teams' ISs due to incompatibilities in infrastructure and difficulty in filtering and validating the typical flood of information generated during disaster events. For example, inconsistency in alert notices' type and format will delay intervention and hinder warnings by fuelling a 'cry wolf' situation where the population is saturated with ambiguous and/or irrelevant messages $[11,12]$. This may lead to sub-optimal prevention and response by intended recipients and potential property and life loss. Efforts to standardise warning message protocols are currently rather localised with low take-up rates [13].

Two main approaches are used to address the above-mentioned problems; they involve either centralisation (hierarchical command) or federalisation of emergency services. Irrespective of the approach used however, proper emergency response and cooperation has still not been achieved, as reflected in criticism expressed in various literature (e.g. [14-16]). The use of military operations-style network-enabled capabilities as the backbone of disaster management [17] is valid only as part of the overall disaster management effort and could also promote over-reliance on an infrastructure that may well fail during disaster events.

Various documents, inquiries reviews and reports ([10-12, 15, 16, 18, 19], etc), suggest that the root causes of current shortcomings could in fact be the inadequate information and knowledge flow / quality between the participants' systems $[20,21]$ owing mostly to incompatibilities originating in heterogeneity, lack of trust, organisational confusion and competition fallacies. True and efficient collaboration requires the interoperability of processes, resources and organisational 
cultures of the participants [22, 23], all of which are reflected in their ISs [24].Another aspect addressed to a lesser extent (albeit being an essential factor in the interoperability requirements) appears to be the life cycles of the DMOs, task forces, government agencies, legislation, service providers and disaster event(s).

Analogies with other domains help provide potential solutions. For example, the DMOs' situation resembles that of commercial enterprises that need to cope with a global business environment requiring them to tackle projects beyond their own resources and knowledge. A typical response in this case is for the companies to set up or join so-called Collaborative Networks [25]. Another analogy is that of allied armed forces that prepare to cooperate in crisis situations by employing standardised agreements (to the extent possible) and joint exercises [26].

DMOs may have full time / militarised and voluntary / reserve staff components depending on the geographical location and local legal and administrative situation. Thus, concepts from both commercial and military areas can be used provided proper customisation is performed so as to fit the specific scenario.

\section{Disaster Management Interoperability Approach and Aspects}

Tackling DMO interoperability requires some clarification: What is the required interoperability extent? What components and/or aspects need to interoperate? How can be ensured that all necessary aspects are covered and especially, that interoperability is preserved over time as all participants evolve? Each disaster event is unique; thus, there is no 'one size fits all' (crisis situations) DMO interoperability level. At a minimum, the participating organisations' systems should be compatible, so at least they don't hinder each other's operations (see Fig.1).

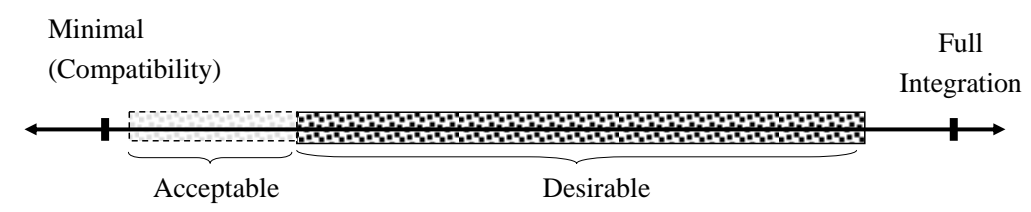

Fig.1 Acceptable and desirable IS Interoperability levels in disaster management

Full integration (if at all achievable) is not desired in this case as it would imply that the DMOs cannot fully function independently. In an emergency situation, some task force members' (information and other) systems may be affected and even cease to function. The other participant DMOs should be able to continue without significant performance loss (see e.g. the ARPANET resilient network concept [27]) and ideally even 'cover' for the participant(s) that failed or is ailing.

Even if a central point of command (e.g. an 'Emergency Command Centre') was secure and unaffected by the disaster event(s), the coordination provided by it 
could be severely impeded by communication breakdown. The remaining task force participants should be able to carry on their duties, at least for a predetermined amount of time. This quasi-independence can be achieved in the form of pre-agreed procedures and shared knowledge, set up well in advance and then continuously monitored and maintained for relevance and consistency.

Referring to the interoperability body of knowledge, ISO14258 [28] establishes several ways to achieve (IS and general) interoperability: integrated (common format for all models), unified (common format at meta level) and federated (participants negotiating an ontology 'on the fly' so as the meaning of models is understood the same way). In the case of DMOs, full integration and federalisation did not seem to succeed and achieve the desired results due to organisational heterogeneity of DMOs and the impossibility to properly negotiate in the limited time available in the case of a disaster event. The seemingly more suitable unified approach assumes that ontology is negotiated in advance; although there have been numerous ontology integration efforts and advances, in disaster management currently there seems to be no effective substitute to DMOs 'spending time together' in order to agree on meanings associated with the concepts used to exchange knowledge. Once that is achieved, proper semantic interoperability should be faster and easier to achieve in the task forces formed by the participant DMOs.

Of all the interoperability aspects to be considered in disaster management, data and process areas appear to be the most urgent (see Fig. 3, right). Thus, the ability to extract and exchange data from heterogeneous sources (delivering high volume and often unreliable data during disaster events) is paramount to being aware of the conditions on the ground and avoiding potentially life-threatening situations for emergency crews and population. Prior agreements on data format and especially on its meaning are essential. Process interoperability here concerns the capability to perform joint operations but also to 'take over' and perform processes on behalf of a disaster management task force participant that may have been temporarily or permanently disabled.

The pragmatic interoperability aspect as described by Whitman and Panetto [5] relates to the willingness and capacity of the participants to interoperate and suggests once again that the human component of the IS needs attention prior to task force formation as to allow gaining trust and knowledge of the other participants.

Organisational interoperability is an important aspect in disaster management as task force participants may often exhibit significant organisational structure diversity. The issues identified by Chen [29] based on the Enterprise Interoperability Framework (EIF), namely responsibility, authority and type of organisation can all impact heavily on the functionality of the disaster management task force. In a crisis situation, the roles (mapping of the human resources onto the decisional structure) and hierarchy must be clear to everyone from the start so that the task force can focus on managing the disaster event rather than spend critical time figuring out its own modus operandi (who does what, who is in charge etc).

Finally, cultural interoperability [5] appears to be one of the hardest obstacles to overcome. The only current working solution appears to be regular immersion of the participant organisations in each other's cultures (e.g. Army joint exercises 
and the expatriate experience of most proficient language translators'). This facilitates the transfer and conversion of tacit and explicit knowledge between the participants, which should be one of the most important goals of interoperability.

The leitmotif running through all aspects analysed above is the 'co-habitation' of the organisations that are expected to form disaster management task forces, as a prerequisite towards the achievement of IS interoperability - whether functional, informational, organisational or cultural. Therefore, this paper proposes the adaptation (in a life cycle context) of the commercial-based Collaborative Network and associated concepts to assist IS interoperability for disaster management.

\section{Collaborative Networks for Disaster Management}

Collaborative Networks (CNs) are created in order to act as 'breeding environments' (BEs) for Virtual Organisations (VOs). A VO is a group of companies that act as one for a limited time in order to bid for, win and complete projects requiring combined resources and know-how; at the end of the project(s) the VO usually dissolves. BE partners may take part in one or several VOs at any given time. A CN 'lead partner' may also be present - elected on size, influence, resources, etc.

The CN concept requires some customisation to make it suitable for the disaster management area. Thus, the time available for set-up of a VO (more suitably hereafter called Disaster Management Task Force - DMTF) is significantly shorter than that available for a project bidding process. In addition, the 'Disaster Management' Collaborative Network (DMCN), its participants and the DMTF(s) produced will operate under tighter, legislated operational guidelines set by the relevant Governments and national / international frameworks.

The commercial and competitive motivations of the typical CN participants will translate in this case into an efficiency and interoperability motivation reflected in lives and property rescued. The usual create / join / remain / leave the $\mathrm{CN}$ decision processes would be mandated from outside (or by the lead partner) for most participants. Reference models (such as patterns) are to be created from lessons learned in past disasters prevention / relief efforts and used to refine future DMTFs. The reference models should be stored in a web-enabled, shared repository and classified on type and location (e.g. flooding, tornadoes, wildfires, severe storms in Australia [30]) and customised to allow for specific intensity, duration, side-effects and consequences.

The DMTF(s) produced by the DMCN will not bid for projects, but rather be set up for and assigned a specific one - the management of a particular disaster event (or combination thereof). The IS management, communication infrastructure, and other organisational interoperability issues would have been sorted out in advance within the DMCN, ensuring a prompt and appropriate task force response and thus addressing a frequent weakness of past crisis management efforts.

Human-related aspects requiring time such as trust and local / organisational culture (including recognition which features prominently in volunteer-based or- 
ganisations [31, 32]) could be also tackled using the concept of an 'emergency services academy' with local branches providing training based on a unique national / interstate curriculum (see [33] for an early initiative).

\section{The Life Cycle Context and Enterprise Architecture}

All entities, including those involved in a disaster management effort, have gone (and will also go) through a series of life cycle phases, possibly several times (e.g. during re-engineering). According to [39], these phases form the entities' life histories. IS interoperability requirements are inherently linked to life history as they will vary during each IS and business life cycle phase; therefore, it is essential that the analysis and search for interoperability improvements is performed in a life cycle context. It is hereby argued that an optimal way to integrate the life cycle aspect into IS interoperability is by using an 'enterprise architecture' (EA) approach.

While there are many definitions for EA, the author takes the commonly accepted view that it expresses the ontology of enterprise change. Thus, EA provides a holistic approach to business evolution and agility "by creating, communicating and improving the key requirements, principles and models that describe the enterprise's future state.” [34] Importantly, “[...] EA comprises people, processes, information and technology of the enterprise, and their relationships to one another and to the external environment” [Ibid.]. This EA definition is in agreement with the view of IS as a socio-technical system [35] with voluntaristic people [38] in a complex organisational, political and behavioural context [36, 37]. As such, it encourages the use of EA as an integrated, multi-perspective approach for analysing the impact of the interactions between the disaster management participants on their individual and task force IS interoperability.

To illustrate the way EA artefacts and methods can guide and enrich the analysis and improvement of DMO interoperability, we have selected a generic architecture framework (AF) subsuming and abstracting several other mainstream AFs that is defined in Annex A of ISO15704 [39] and called Generalised Enterprise Architecture and Methodology (GERAM). The modelling framework (MF) of GERAM's reference architecture (GERA) contains a rich repository of aspects (including human) that can all be represented in a life cycle context. GERA's MF has been previously used in IS management modelling and other tasks (e.g. see $[40,41])$.

Subsets of the GERA MF can be used to build life cycle-based formalisms such as shown in Fig. 2, top. These can then be utilised in the creation of business models requiring a life cycle background. Thus, aspects previously identified as significant in tackling IS disaster management interoperability (function, information, organisation, human aspect) can be represented together as shown in Fig. 2 upper left. Aspects can also be separated (e.g. for clarity) using a 'flattened' representation; for example, the 2-dimensional structure shown in Fig. 2 upper right can be used for separate diagrams for information, function, and so on. 


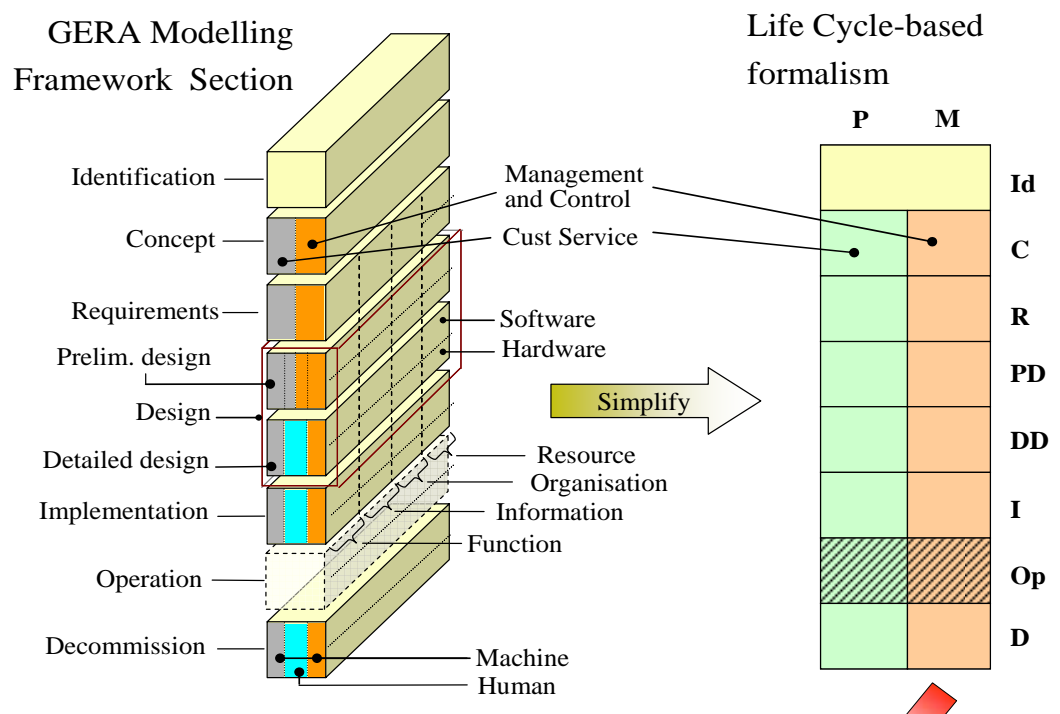

Disaster Event modelling

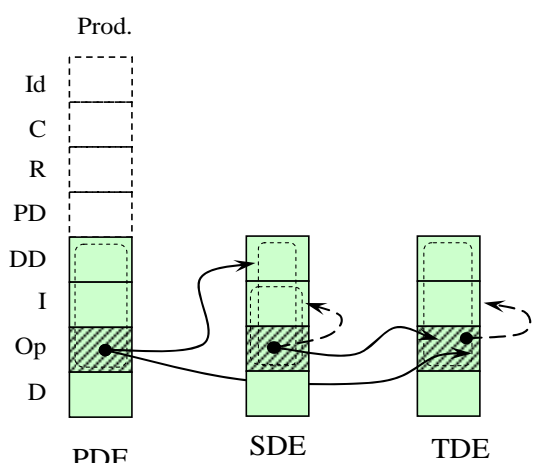

Disaster Event mapping

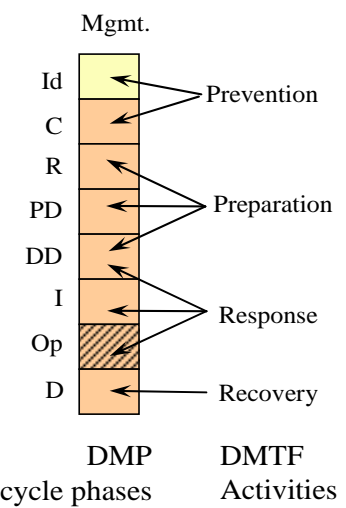

Legend:

PDE / SDE / TDE = Primary / Secondary / Tertiary Disaster Event;

DMP = Disaster Mgmt Project; DMTF = Disaster Management Task Force

$\square=$ Production view $\square=$ Management view $\bullet \rightarrow=$ Possible scenario

Fig. 2 Disaster event modelling and mapping using the GERA life cycle formalism

Fig. 2 lower left uses the GERA-based modelling formalism to show a high-level representation of a disaster event, taking into account its relevant life cycle phases and possible relations to other events. Thus, it can be conveyed for example that a Primary Disaster Event (PDE) can trigger / influence other events (SDE, TDE). For example, an earthquake event (PDE) can trigger a tsunami (SDE) that can in turn trigger a partial nuclear meltdown (TDE). However PDE can also influence 
TDE directly. Events such as chain reactions can also be shown (arrows from Operation to Implementation within same entity). Here, the GERA MF's 'Detailed Design' and 'Implementation' life cycle phases refer to features of the event - e.g. earthquake time, epicentre and duration or tsunami spread, wave speed, height etc.

The lower right hand side of Fig. 2 shows how the life cycle phases of a disaster management project (DMP) can be mapped to the typical disaster management activities [8] performed by the DMTF that sets up and operates that project. Such diagrams are useful to help stakeholders grasp a common understanding of the disaster event and management aspects, thus overcoming some IS interoperability semantic barriers that may delay DMP kick-off and thus hinder DMTF response.

Fig. 3 uses a modified version of Chen's EIF (used in Section 3 to identify types, aspects and barriers to interoperability) and GERA's MF-based life cycle modelling formalism to show how the relevance and applicability of the IS interoperability aspects depend on the specific life cycle phases of the disaster event and the DMTFs' actions to address them. The figure shows that different IS interoperability aspects take precedence during various disaster event life cycles.

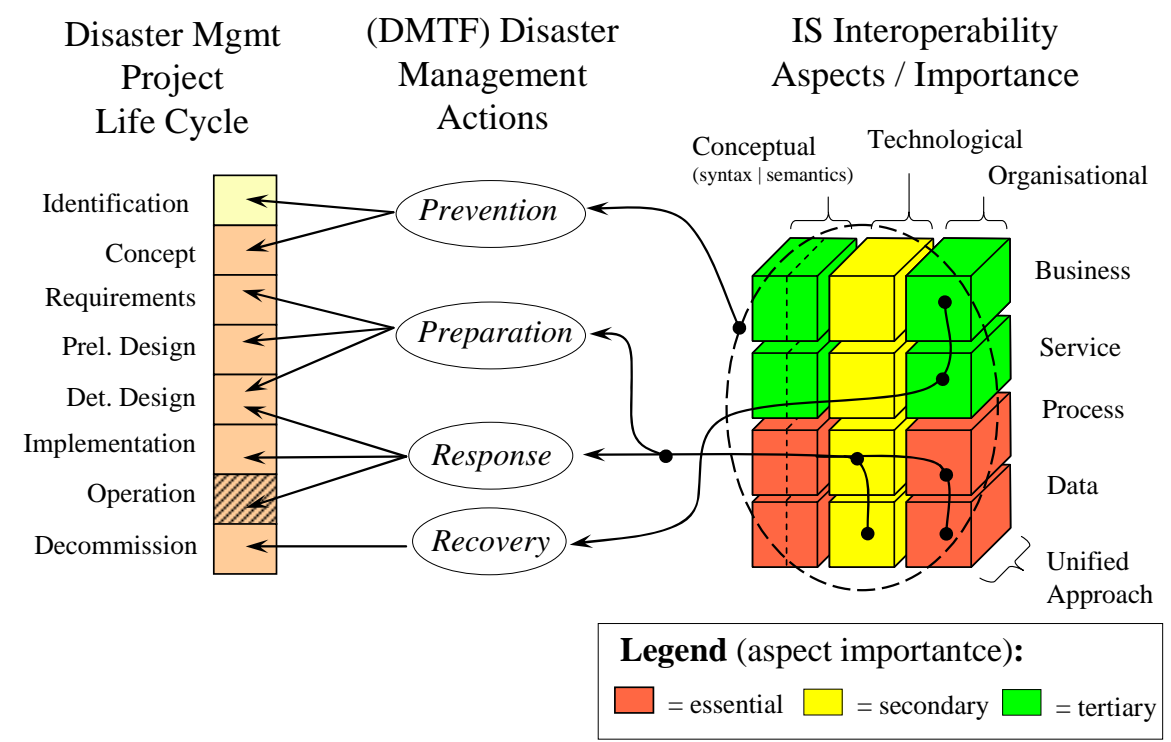

Fig. 3 IS Interoperability requirements mapped on disaster management actions and the disaster management project life cycle, using Chen's EIF [29] and GERA's MF-based formalism

For example, during the response phase, data and process IS interoperability aspects are paramount as accurate and fresh data is required and processes may need to be performed interchangeably due to potential disability of DMTF participants (or need to involve replacement DMCN members). During the recovery, which typically takes place over an extended period of time and requires less real-time responsiveness, business and service IS interoperability aspects take precedence. 


\section{Application to IS Data Interoperability: A Possible Scenario}

Although very helpful in setting up the task force and DM project, Fig. 3 shows only the influence of the disaster event, management project and task force life cycles on the interoperability requirements. As a matter of fact, the DMOs, DMTF and DMP interact with a plethora of entities and artefacts during their life history. These interactions must also to be analysed in order to gain a whole-system view of the IS interoperability endeavour.

Using the GERA-based formalism shown in Fig. 2, several diagrams may be built for the aspects identified in Section 3 as important to IS interoperability. For example, Fig. 4 shows interoperability-related interactions for the Information (data) aspect of the GERA MF, in the previously described scenario of a breeding environment (DMBE) creating task forces (DMTFs) supervising disaster management projects (DMPs) that coordinate disaster event responses.

The arrows in the figure show data interoperability requirements and influences. For example, the participating DMOs' ISs need to interoperate during their Operation life cycle phase; however this may also imply some redesign (line between DMO1 and DMO2 linking Operation but also 'upper' life cycle phases). The IS of the DMBE must be able to interoperate with the IS of the DMTF it creates and DMPs managed by them in their Operation life cycle phases, with influences on other phases in case of subsequent redesign (details omitted for clarity).

In another example, data 'interoperation' of the population POP with the Government's Disaster Management Agencies (GDMAs, such as [42]) is important as may result in changes to legislation - hence in the DMO and DMBE interoperability requirements. However, data interoperability between the ISs of DMOs participating in a DMTF and between the DMTF's IS and POP is paramount as it will directly influence the extent of lost property and casualties. Past (and sometimes tragic) experience and feedback from subsequent enquiries [11, 12, 30] has shown that two major goals of the disaster management interoperability improvement effort (partly reflected in Fig. 4) should be a) whether POP receives, understands, believes and acts on DMTF warnings and directives and b) that DMTF participants can properly interoperate during the disaster event. Thus the IS of the DMTFs (and implicitly DMOs) should be also designed to avoid ambiguity and maximise focus in relation to the local specific semantic interoperability requirements (language, technology type, habits etc). For example, the message format and distribution in a densely populated and developed area would most likely differ from the one used in a sparsely and/or underdeveloped region - at least until efforts to standardise warnings are successful, widely implemented and observed.

Importantly, some organisations shown in Fig. 4 should be able to redesign themselves to a certain extent (arrows from Operation life cycle phase to upper phases within same entities e.g. in DMBE, DMO, DMTF). This reflects an essential capability to adapt (and thus be agile and resilient) in the face of changes in the situation and environment that are likely to occur briskly and unexpectedly during disaster events. 
The Disaster Management Qualification and Training organisation (DMQT, see e.g. [33]) can assess the suitability of organisations to enter a DMBE by requiring (and providing training if necessary towards) IS data interoperability between DMO applicants and the DMBE. This requirement may also go beyond the Operation life cycle phase should training / redesign of the participants be performed.

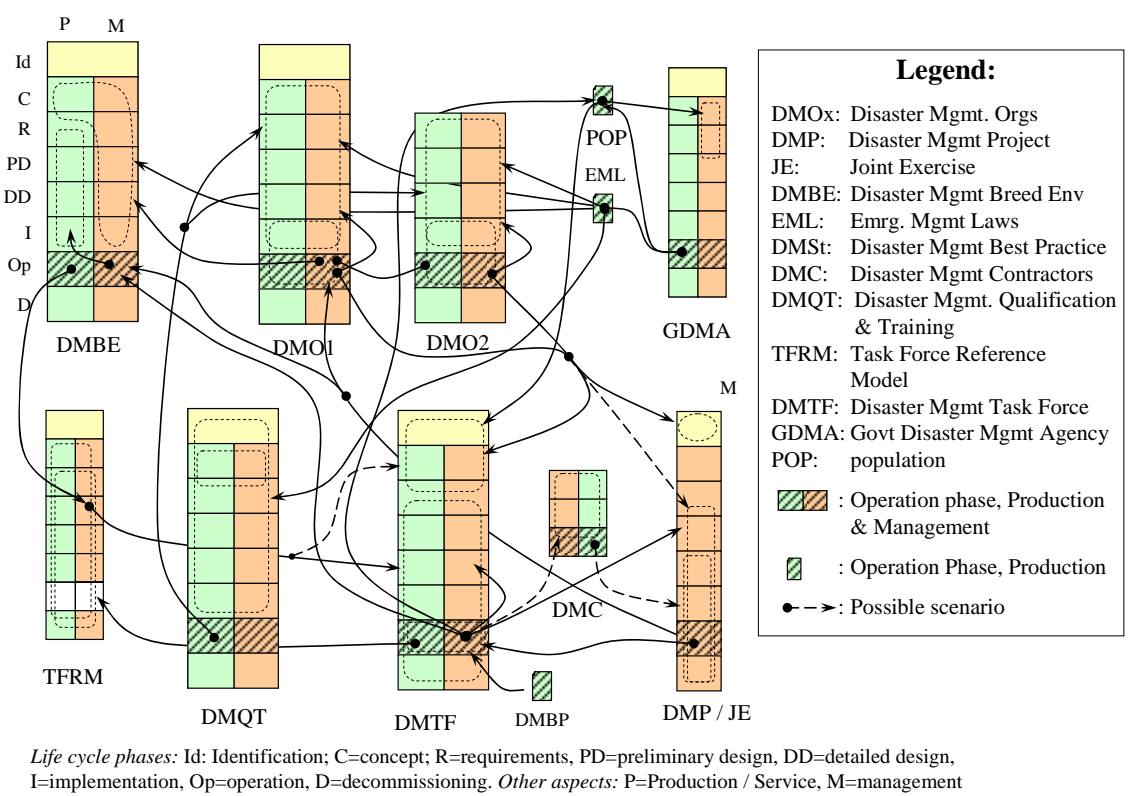

Fig. 4. High-level IS Data Interoperability requirements in the context of a Disaster Management Breeding Environment and Task Force solution (details omitted for clarity)

The validity and effectiveness of the DMBE concept can be tested by joint exercises (JEs) simulating disaster events; this will also allow practical and potentially hidden IS data interoperability problems to surface and be tackled. Importantly, the resilience of DMTFs created can be also assessed in JEs through various scenarios and proper corrective action be taken to improve DMOs' preparedness.

\section{Conclusions and Further Work}

Disaster management interoperability-related issues are best addressed in advance and in a holistic manner so that when a disaster event occurs an efficient, collaborative task force can be promptly put together. Therefore,, in order to tackle the IS interoperability aspects relevant to disaster management identified and prioritised using state-of the-art research in the domain, it is proposed to adopt the commercial breeding environment and virtual enterprise concepts, customised for disaster 
management. In addition, as the life cycle of the disaster management participants plays a central role in the IS interoperability requirements, it is argued that (and shown how) an EA approach can provide an integrated, life cycle context to the entire disaster management IS interoperability improvement effort.

Further research will concentrate on testing, verifying and validating the findings with DMO stakeholders within several past, present and future case studies.

\section{References}

1. Elkington, J., Cannibals with Forks: The Triple Bottom Line of $21^{\text {st }}$ Century Business. 1998.

2. Blackburn, W.R., The Sustainability Handbook. 2007, Cornwall, UK: EarthScan Publishers.

3. UN World Commission on Environment and Development, Our Common Future (Brundtland Report). 1987, Oxford: Oxford University Press.

4. Noran, O., Integrating Environmental and Information Systems Management: An Enterprise Architecture Approach, in Information Systems Development: Asian Experiences (Proceedings of the $18^{\text {th }}$ International Conference on Information Systems Development (ISD 2009)), W. Song, et al., Editors. 2011, Springer Verlag: Nanchang, China. p. 123-134.

5. Whitman, L. and H. Panetto, The Missing Link: Culture and Language Barriers to Interoperability. Annual Reviews in Control, 2006. 30(2): p. 233-241.

6. Lehtinen, E. and K. Lyytinen, Action based model of information system. Information Systems Research, 1986. 11(4): p. 299-317.

7. Government of South Australia. Emergency Management Act 2004. 2004 [cited 2011 Mar 30]; Available from: http://www.legislation.sa.gov.au/LZ/C/A/EMERGENCY MANAGEMENT ACT 2004.aspx.

8. Australian Government. Attorney's General's Office - Emergency Management in Australia. 2011 [cited 2011 Mar 30]; Available from: http://www.ema.gov.au/.

9. Federal Emergency Management Agency. National Response Framework. 2011 [cited 2011 Mar 30]; Available from: http://www.fema.gov/pdf/emergency/nrf/about_nrf.pdf.

10. United Nations International Strategy for Disaster Reduction Secretariat (UNISDR). Hyogo Framework for Action 2005-2015: Building the resilience of nations and communities to disasters. 2011 [cited 2011 Mar 30]; Available from:

http://www.preventionweb.net/files/1037_hyogoframeworkforactionenglish.pdf.

11. Queensland Floods Commission of Enquiry. Submissions to the Enquiry. 2011 [cited 2011 July]; Available from: http://www.floodcommission.qld.gov.au/submissions/.

12. Victorian Bushfires Royal Commission. Submissions to the Enquiry. 2009 [cited 2011 July]; Available from: http://www.royalcommission.vic.gov.au/Submissions/View-Submissions.

13. OASIS. Common Alerting Protocol v1.1. 2005 [cited 2012; Available from: http://www.oasisopen.org/committees/download.php/15135/emergency-CAPv1.1-Corrected_DOM.pdf.

14. Clark, J.L., Practical aspects of federalizing disaster response. Critical Care, 2006. 10: p. 107-113.

15. Wiese, C.R., Organizing Homeland Security after Katrina: is adaptive management what's missing? Public Administration Review, 2006. 66(3): p. 302-318.

16. 't Hart, P., et al., The Politics of Crisis Management: Public Leadership under Pressure. Journal of Homeland Security and Emergency Management. 2005, Cambridge UK: Cambridge University Press.

17. von Lubitz, D.K.J.E., J.E. Beakley, and F. Patricelli, Disaster Management: The Structure, Function, and Significance of Network-Centric Operations. Journal of Homeland Security asnd Emergency Management, 2008. 5(1): p. Art 42.

18. Brewin, B. Tsunami response reveals poor radio interoperability. 2011 [cited 2012 April]; Available from: http://www.nextgov.com/nextgov/ng_20110415_3972.php. 
19. Igarashi, Y., et al., Anatomy of Historical Tsunamis: Lessons Learned for Tsunami Warning. Pure Applied Geophysics, 2011(168): p. 2043-2063.

20. Wickramasinghe, N. and D.K.J.E. von Lubitz, Knowledge Based Enterprises: Theories and Fundamentals. Journal of Homeland Security asnd Emergency Management. Vol. 5. 2007, Hershey PA: IGP Publishing Group,.

21. Prizzia, R. and G. Helfand, Emergency preparedness and disaster management in Hawaii. Disaster Prevention and Management, 2001. 10(3): p. 163-172.

22. Kapucu, N., T. Arslan, and F. Demiroz, Collaborative emergency management and national emergency management network. Disaster Prevention \& Management, 2010. 19(4): p. 452-468.

23. Trakas, A. Interoperability - A key requirement for emergency and disaster management. 2012 [cited 2012; Available from: http://www.un-spider.org/book/5143/4c-challenge-communicationcoordination-cooperation-capacity-development.

24. Stohr, E. and B.R. Konsynsky, Information Systems and Decision Processes. IEEE Computer Society Press. 1992, California.

25. Camarinha-Matos, L., et al., Collaborative networked organizations - Concepts and practice in manufacturing enterprises. Computers and Industrial Engineering, 2009. 57(1): p. 46-60.

26. NATO. Interoperability for joint operations. 2006 [cited 2011 July]; Available from: http://www.nato.int/docu/interoperability/interoperability.pdf.

27. Heart, F., et al., ARPANET Completion Report, Bolt, Beranek, and Newman, Editors. 1978: Burlington, MA.

28. ISO, ISO14258 Industrial Automation Systems - Concepts and Rules for Enterprise Models. 2005.

29. Chen, D. Framework for Entrerprise Interoperability. 2006 [cited 2011 July]; Available from: http://www.fines-cluster.eu/fines/jm/Download-document/53-Framework-for-EnterpriseInteroperability-Chen.html.

30. Ellis, S., P. Kanowski, and R. Whelan, National inquiry into bushfire mitigation and management. 2004, Commonwealth of Australia: Canberra.

31. Esmond, J. Report on The Attraction, Support and Retention of Emergency Management Volunteers. 2011 [cited 2011 Mar 30]; Available from: http://www.ema.gov.au/www/emaweb/emaweb.nsf/Page/Volunteers2.

32. McLennan, J. Issues Facing Australian Volunteer-Based Emergency Services Organisations: 2008 -2010. 2008 [cited 2011 Mar 30]; Available from: http://www.ema.gov.au/www/emaweb/emaweb.nsf/Page/Volunteers2.

33. Queensland Government. Queensland Combined Emergency Services Academy (QCESA). 2011 [cited 2011 Mar 30]; Available from: http://www.safecom.sa.gov.au/site/emergency_management.jsp.

34. Gartner Research. IT Glossary. 2012 [cited 2012; Available from http://www.gartner.com/technology/it-glossary/enterprise-architecture.jsp.

35. Pava, C., Managing New Office Technology, An Organisational Strategy. 1983, New York: Free Press.

36. Keen, P.G.W. and M. Scott Morton, Decision Support Systems: An Organisational Perspective. 1978, Reading, Massachussetts: Addison-Wesley.

37. Iivari, J., A Paradigmatic Analysis of Contemporary Schools of IS Development. Eur. J. Information Systems, 1991. 1(4): p. 249-272.

38. McGregor, D., The Human Side of Enterprise. 1960, New York: McGraw-Hill.

39. ISO/IEC, Annex C: GERAM, in ISO/IS 15704:2000/Amd1:2005: Industrial automation systems Requirements for enterprise-reference architectures and methodologies. 2005.

40. Noran, O., A Meta-methodology for Collaborative Networked Organisations: Creating Directly Applicable Methods for Enterprise Engineering Projects. 2008, Saarbrücken: VDM Verlag Dr. Müller.

41. Noran, O., Engineering the Sustainable Business: An Enterprise Architecture Approach, in Coherency Management: Architecting the Enterprise for Alignment, Agility, and Assurance, G. Doucet, J. Gotze, and P. Saha, Editors. 2009, International Enterprise Architecture Institute. p. 179-210.

42. Government of Queensland. Department of Community Safety - Structure. 2012 [cited 2012 Apr]; Available from: http://www.emergency.qld.gov.au/about/. 